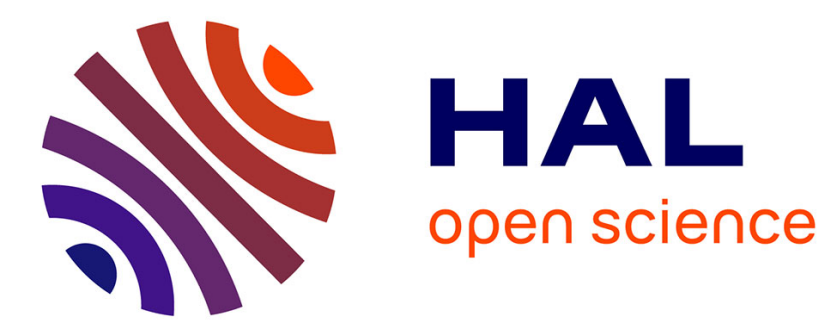

\title{
Expressing Additives Using Multiplicatives and Subexponentials
}

Kaustuv Chaudhuri

\section{To cite this version:}

Kaustuv Chaudhuri. Expressing Additives Using Multiplicatives and Subexponentials. Mathematical Structures in Computer Science, 2018, 28 (5), pp.651-666. hal-01222767

HAL Id: hal-01222767

https://hal.inria.fr/hal-01222767

Submitted on 30 Oct 2015

HAL is a multi-disciplinary open access archive for the deposit and dissemination of scientific research documents, whether they are published or not. The documents may come from teaching and research institutions in France or abroad, or from public or private research centers.
L'archive ouverte pluridisciplinaire $\mathbf{H A L}$, est destinée au dépôt et à la diffusion de documents scientifiques de niveau recherche, publiés ou non, émanant des établissements d'enseignement et de recherche français ou étrangers, des laboratoires publics ou privés.

\section{()ㅜ) $\Theta$}

Distributed under a Creative Commons Attribution - NoDerivatives| 4.0 International 


\title{
Expressing Additives Using Multiplicatives and Subexponentials
}

\author{
Kaustuv Chaudhuri \\ Inria \& LIX/École polytechnique, France \\ kaustuv.chaudhuri@inria.fr
}

17 August 2015

\begin{abstract}
Subexponential logic is a variant of linear logic with a family of exponential connectives - called subexponentials - that are indexed and arranged in a pre-order. Each subexponential has or lacks associated structural properties of weakening and contraction. We show that a classical propositional multiplicative subexponential logic (MSEL) with one unrestricted and two linear subexponentials can encode the halting problem for two register Minsky machines, and is hence undecidable. We then show how the additive connectives can be directly simulated by giving an encoding of propositional multiplicative additive linear logic $(M A L L)$ in an MSEL with one unrestricted and four linear subexponentials.
\end{abstract}

\section{Introduction}

The decision problem for classical propositional multiplicative exponential linear logic (MELL), consisting of formulas constructed from propositional atoms using the connectives $\{\otimes, 1, \not \supset, \perp, !, ?\}$, is perhaps the longest standing open problem in linear logic. MELL is bounded below by the purely multiplicative fragment (MLL), which is decidable even in the presence of first-order quantification, and above by MELL with additive connectives (MAELL), which is undecidable even for the propositional fragment []ㅡ. This paper tries to make the undecidable upper bound a bit tighter by considering the question of the decision problem for a family of propositional multiplicative subexponential logics (MSEL) [10, 11], each of which consists of formulas constructed from propositional atoms using the (potentially infinite) set of connectives $\{\otimes, 1, \ngtr,, \perp\} \cup \bigcup_{u \in \Sigma}\left\{!^{u}, ?^{u}\right\}$, where $\Sigma$ is a pre-ordered set of subexponential labels, called a subexponential signature, that is a parameter of the family of logics. In particular, we show that a particular MSEL with a subexponential signature consisting of exactly three labels can encode a two register Minsky machine (2RM), which is Turing-equivalent. This is the same strategy used in [6] to show the undecidability of MAELL, but the encoding in MSEL is different - simpler - for the branching instructions, and shows that additive behavior is not essential to implement testing for zero, which is the operator that appears to be difficult - likely impossible - to encode in ordinary MELL.

This simple demonstration of undecidability raises an obvious proof-theoretic question: are the additive connectives redundant with multiplicatives and subexponentials? Recall that, with the usual unrestricted exponential connectives of MAELL, that certain compound connectives with additive sub-components can be equivalently expressed without the additive connectives: $!(A \& B) \equiv ! A \otimes ! B$ and $?(A \oplus B) \equiv ? A \ngtr ? B$. The standard proofs of these equivalences make use of the contraction and weakening rules for ?, and are therefore not suitable for the situation where ! and ? do not enjoy these structural properties. Nevertheless, these equivalences encode an essential insight about how they may be implemented. To illustrate, if we were able to interpret the ! connective as a label for the current context, then the implication $! A \otimes ! B \multimap !(A \& B)$ can be read as:

To prove $A \& B$ in a given context, separately prove $A$ and $B$ each in a copy of the context.

We will show how the subexponential connectives can be used to build the operation of copying a context. We use a fairly obvious strategy: to copy a context we need to run a computation that duplicates each element of the context until quiescence, that is, until every available formula has been copied. This much can be done with ordinary linear logic. What subexponentials add is the ability to detect when the copying computation is finished, and then, and only then, to progress to the next step. The full MALL proof system is encoded in terms of these staged quiescent computations.

This short paper is organized as follows: in section $\underline{2}$ we sketch the one-sided sequent formulation of MSEL and recall the definition of a $2 R M$. In section $\underline{3}$ we encode the transition system of a $2 R M$ in a MSEL with a particular signature. In section $\underline{4}$ we argue that the encoding is adequate, i.e., that the halting problem for a $2 R M$ is reduced to the proof search problem for this MSEL-encoding, by appealing to a focused sequent calculus 


$$
\begin{aligned}
& \overline{\vdash a, \neg a}_{\text {init }} \frac{\vdash \Gamma, A \quad \vdash \Delta, B}{\vdash \Gamma, \Delta, A \otimes B} \otimes \overline{\vdash 1}^{1} \quad \frac{\vdash \Gamma, A}{\vdash \Gamma, A \oplus B} \oplus_{1} \frac{\vdash \Gamma, B}{\vdash \Gamma, A \oplus B} \oplus_{2} \quad \text { no rule for 0 } \\
& \frac{\vdash \Gamma, A, B}{\vdash \Gamma, A \not 8 B} \ngtr \quad \frac{\vdash \Gamma}{\vdash \Gamma, \perp} \perp \frac{\vdash \Gamma, A \quad \vdash \Gamma, B}{\vdash \Gamma, A \& B} \& \quad \frac{\vdash \Gamma, \top}{1}^{\top} \frac{\vdash \Gamma, A}{\vdash \Gamma, ?^{u} A} ? \\
& \frac{\left(u \leq_{\Sigma} \vec{v}\right) \vdash_{\Sigma} ?^{\vec{v}} \vec{A}, C}{\vdash_{\Sigma} ?^{\vec{v}} \vec{A}, !^{u} C} ! \quad \frac{\left(u \in U_{\Sigma}\right) \vdash_{\Sigma} \Gamma}{\vdash_{\Sigma} \Gamma, ?^{u} A} \text { weak } \quad \frac{\left(u \in U_{\Sigma}\right) \vdash_{\Sigma} \Gamma, ?^{u} A, ?^{u} A}{\vdash_{\Sigma} \Gamma, ?^{u} A} \text { contr }
\end{aligned}
$$

Figure 1: Inference rules for a cut-free one-sided sequent calculus formulation of SEL. Only the rules on the last line are sensitive to the signature.

for MSEL. Then, in section $\underline{5}$ we give an encoding of $M A L L$ in a different instance of $M S E L$, and show that it adequately captures MALL proofs. The final section $\underline{6}$ discusses some consequences.

Note: We use the classical dialect of linear logic to show these results. The intuitionistic dialect has the same decision problem because it is possible to faithfully encode (i.e., linearly simulate the sequent proofs of) the classical dialect in the intuitionistic dialect without changing the signature [2]. This paper is an extended version of []].

\section{Background}

\subsection{Propositional Subexponential Logic}

Let us quickly recall propositional subexponential logic (SEL) and its associated sequent calculus proof system. This logic is sometimes called subexponential linear logic (SELL), but since it is possible for the subexponentials to have linear semantics it is redundant to include both adjectives. Formulas of $S E L(A, B, \ldots)$ are built from atomic formulas $(a, b, \ldots)$ according to the following grammar:

$$
\begin{aligned}
& A, B, \ldots::=a|A \otimes B| 1|A \oplus B| 0 \mid !^{u} A \\
& |\underset{\text { atomic }}{\neg a \mid}| \underset{\text { multiplicative }}{A \not B}|\perp| A \& B|\top| ?^{u} A
\end{aligned}
$$

Each column in the grammar above is a De Morgan dual pair. A positive formula (depicted with $P$ or $Q$ when relevant) is a formula belonging to the first line of the grammar, and a negative formula (depicted with $N$ or $M)$ is a formula belonging to the second line. The labels $(u, v, \ldots)$ on the subexponential connectives $!^{u}$ and $?^{u}$ belong to a subexponential signature defined below. The additive fragment of this syntax is just used in this section for illustration; we will not be using the additives in our encodings. The fragment without the additives will be called multiplicative subexponential logic (MSEL).

Definition 1. A subexponential signature $\Sigma$ is a structure $\langle\Lambda, U, \leq\rangle$ where:

- $\Lambda$ is a countable set of labels;

- $U \subseteq \Lambda$, called the unbounded labels; and

- $\leq \subseteq \Lambda \times \Lambda$ is a pre-order on $\Lambda$ - i.e., it is reflexive and transitive - and $\leq$-upwardly closed with respect to $U$, i.e., for any $u, v \in \Lambda$, if $u \in U$ and $u \leq v$, then $v \in U$.

We will assume an ambient signature $\Sigma$ unless we need to disambiguate particular instances of $M S E L$, in which case we will use $\Sigma$ in subscripts. For instance, $M S E L_{\Sigma}$ is a particular instance of $M S E L$ for $\Sigma$.

The true formulas of MSEL are derived from a sequent calculus proof system consisting of sequents of the form $\vdash A_{1}, \ldots, A_{n}$ (with $n>0$ ) and abbreviated as $\vdash \Gamma$. The contexts $(\Gamma, \Delta, \ldots)$ are multi-sets of formulas of SEL, and $\Gamma, \Delta$ and $\Gamma, A$ stand as usual for the multi-set union of $\Gamma$ with $\Delta$ and $\{A\}$, respectively. The inference rules for $S E L$ sequents are displayed in figure 1 . Most of the rules are shared between $S E L$ and linear logic and will not be elaborated upon here. The differences are with the subexponentials, for which we use the following definition.

Definition 2. For any $n \in N$ and lists $\vec{u}=\left[u_{1}, \ldots, u_{n}\right]$ and $\vec{A}=\left[A_{1}, \ldots, A_{n}\right]$, we write $?^{\vec{u}} \vec{A}$ to stand for the context $?^{u_{1}} A_{1}, \ldots, ?^{u_{n}} A_{n}$. For $\vec{v}=\left[v_{1}, \ldots, v_{n}\right]$, we write $u \leq \vec{v}$ to mean that $u \leq v_{1}, \ldots$, and $u \leq v_{n}$.

The rule for !, sometimes called promotion, has a side condition that checks that the label of the principal formula is less than the labels of all the other formulas in the context. This rule cannot be used if there are non-?-formulas in the context, nor if the labels of some of the ?-formulas are strictly smaller or incomparable 
with that of the principal !-formula. Both these properties will be used in the encoding in the next section. The structural rules of weakening and contraction apply to those principal ?-formulas with unbounded labels.

\subsection{Two Register Minsky Machines}

Like Turing machines, Minsky register machines have a finite state diagram and transitions that can perform $\mathrm{I} / \mathrm{O}$ on some unbounded storage device, in this case a bank of registers that can store arbitrary natural numbers. We shall limit ourselves to machines with two registers $(2 R M)$ a and $b$, which are sufficient to encode Turing machines.

Definition 3. A $2 R M$ is a structure $\langle Q, *, \mathcal{C}, \rightarrow\rangle$ where:

- $Q$ is a non-empty finite set of states;

- $* \in Q$ is a distinguished halting state;

- $\mathcal{C}$ is a set of configurations, each of which is a structure of the form $\langle q, v\rangle$, with $q \in Q$ and $v:\{\mathrm{a}, \mathrm{b}\} \rightarrow N$, that assigns values (natural numbers) to the registers a and $\mathrm{b}$ in state $q$;

- $\rightarrow \subseteq \mathcal{C} \times I \times \mathcal{C}$ is a deterministic labeled transition relation between configurations where the label set $I=\{$ halt, incra, incrb, decra, decrb, isza, iszb $\}$ (called the instructions).

By usual convention, we write $\rightarrow$ infix with the instruction atop the arrow. We require that every element of $\longrightarrow$ fits one of the following schemas, where in each case $q, r \in Q$ and $q \neq r$ :

$$
\begin{aligned}
& \langle q, v\rangle \stackrel{\text { halt }}{\longrightarrow}\langle *,\{\mathrm{a}: 0, \mathrm{~b}: 0\}\rangle \quad(\text { with } q \neq *) \\
& \langle q,\{\mathrm{a}: m, \mathrm{~b}: n\}\rangle \stackrel{\text { incra }}{\longrightarrow}\langle r,\{\mathrm{a}: m+1, \mathrm{~b}: n\}\rangle \\
& \langle q,\{\mathrm{a}: m, \mathrm{~b}: n\}\rangle \stackrel{\text { incrb }}{\longrightarrow}\langle r,\{\mathrm{a}: m, \mathrm{~b}: n+1\}\rangle \\
& \langle q,\{\mathrm{a}: m+1, \mathrm{~b}: n\}\rangle \stackrel{\text { decra }}{\longrightarrow}\langle r,\{\mathrm{a}: m, \mathrm{~b}: n\}\rangle \\
& \langle q,\{\mathrm{a}: m, \mathrm{~b}: n+1\}\rangle \stackrel{\text { decrb }}{\longrightarrow}\langle r,\{\mathrm{a}: m, \mathrm{~b}: n\}\rangle \\
& \langle q,\{\mathrm{a}: 0, \mathrm{~b}: n\}\rangle \stackrel{\text { isza }}{\longrightarrow}\langle r,\{\mathrm{a}: 0, \mathrm{~b}: n\}\rangle \\
& \langle q,\{\mathrm{a}: m, \mathrm{~b}: 0\}\rangle \stackrel{\text { iszb }}{\longrightarrow}\langle r,\{\mathrm{a}: m, \mathrm{~b}: 0\}\rangle
\end{aligned}
$$

For a trace $\vec{i}=\left[i_{1}, \ldots, i_{n}\right]$, we write $\left\langle q_{0}, v_{0}\right\rangle \stackrel{\vec{i}}{\longrightarrow}\left\langle q_{n}, v_{n}\right\rangle$ if $\left\langle q_{0}, v_{0}\right\rangle \stackrel{i_{1}}{\longrightarrow} \cdots \stackrel{i_{n}}{\longrightarrow}\left\langle q_{n}, v_{n}\right\rangle$. The $2 R M$ halts from an initial configuration $\left\langle q_{0}, v_{0}\right\rangle$ if there is a trace $\vec{i}$ such that $\left\langle q_{0}, v_{0}\right\rangle \stackrel{\vec{i}}{\longrightarrow}\langle *,\{\mathrm{a}: 0, \mathrm{~b}: 0\}\rangle$. (The configuration $\langle *,\{\mathrm{a}: 0, \mathrm{~b}: 0\}\rangle$ will be called the halting configuration.) The halting problem for a $2 R M$ is the decision problem of whether the machine halts from an initial configuration.

The requirement that $\rightarrow$ be deterministic amounts to: $\langle q, v\rangle \stackrel{i}{\longrightarrow}\left\langle q_{1}, v_{1}\right\rangle$ and $\langle q, v\rangle \stackrel{j}{\longrightarrow}\left\langle q_{2}, v_{2}\right\rangle$ imply that $i=j, q_{1}=q_{2}$, and $v_{1}=v_{2}$. Note that a trace that does not end with a halting configuration will not be considered to be halting, even if there is no possible successor configuration. It is an easy exercise to transform a given $2 R M$ into one where every configuration has a successor except for the halting configuration.

Theorem 4 ([9]). The halting problem for 2 RMs is recursively unsolvable.

\section{Encoding a $2 R M$}

For a given $2 R M$, which we fix in this section, we will encode its halting problem as the derivability of a particular MSEL sequent that encodes its labeled transition system and the initial configuration. We will use the following subexponential signature in the rest of this section.

Definition 5. Let $\Xi$ stand for the signature $\langle\{\infty, \mathrm{a}, \mathrm{b}\},\{\infty\}, \leq\rangle$ where $\leq$ is the reflexive-transitive closure of $\leq_{0}$ defined by $\mathrm{a} \leq_{0} \infty$ and $\mathrm{b} \leq_{0} \infty$.

Definition 6 (encoding configurations). For $c=\langle q, v\rangle$, we write $\mathcal{E}(c)$ for the following $M S E L \Xi$ context:

$$
\underbrace{?^{\mathrm{a}} \neg \mathrm{ra}, ?^{\mathrm{a}} \neg \mathrm{ra}, \ldots, ?^{\mathrm{a}} \neg \mathrm{ra}}_{\text {length }=v(\mathrm{a})}, \underbrace{?^{\mathrm{b}} \neg \mathrm{rb}, ?^{\mathrm{b}} \neg \mathrm{rb}, \ldots, ?^{\mathrm{b}} \neg \mathrm{rb}}_{\text {length }=v(\mathrm{~b})}, \neg q
$$

Definition 7 (encoding transitions). The transitions $(\underline{1})$ of the $2 R M$ are encoded as a context $\Pi$ with: 
- to represent $\langle q, v\rangle \stackrel{\text { halt }}{\longrightarrow}\langle *,\{\mathrm{a}: 0, \mathrm{~b}: 0\}\rangle$, the elements: $q \otimes \neg \mathrm{h}, \mathrm{h} \otimes !^{\mathrm{a}} \mathrm{ra} \otimes \neg \mathrm{h}, \mathrm{h} \otimes !^{\mathrm{b}} \mathrm{rb} \otimes \neg \mathrm{h}, \mathrm{h} \otimes !^{\infty} 1$ (for some $\mathrm{h} \notin Q)$ :

- to represent $\langle q,\{\mathrm{a}: m, \mathrm{~b}: n\}\rangle \stackrel{\text { incra }}{\longrightarrow}\langle r,\{\mathrm{a}: m+1, \mathrm{~b}: n\}\rangle$, the element $q \otimes\left(\neg r \not ?^{\mathrm{a}} \neg \mathrm{ra}\right)$;

- to represent $\langle q,\{\mathrm{a}: m, \mathrm{~b}: n\}\rangle \stackrel{\text { incrb }}{\longrightarrow}\langle r,\{\mathrm{a}: m, \mathrm{~b}: n+1\}\rangle$, the element: $q \otimes\left(\neg r \not 8 ?^{\mathrm{b}} \neg \mathrm{rb}\right)$;

- to represent $\langle q,\{\mathrm{a}: m+1, \mathrm{~b}: n\}\rangle \stackrel{\text { decra }}{\longrightarrow}\langle r,\{\mathrm{a}: m, \mathrm{~b}: n\}\rangle$, the element: $q \otimes !^{\mathrm{a}} \mathrm{ra} \otimes \neg r$;

- to represent $\langle q,\{\mathrm{a}: m, \mathrm{~b}: n+1\}\rangle \stackrel{\text { decrb }}{\longrightarrow}\langle r,\{\mathrm{a}: m, \mathrm{~b}: n\}\rangle$, the element: $q \otimes !^{\mathrm{b}} \mathrm{rb} \otimes \neg r$;

- to represent $\langle q,\{\mathrm{a}: 0, \mathrm{~b}: n\}\rangle \stackrel{\text { isza }}{\longrightarrow}\langle r,\{\mathrm{a}: 0, \mathrm{~b}: n\}\rangle$, the element: $q \otimes !^{\mathrm{b}} \neg r ;$ and

- to represent $\langle q,\{\mathrm{a}: m, \mathrm{~b}: 0\}\rangle \stackrel{\text { iszb }}{\longrightarrow}\langle r,\{\mathrm{a}: m, \mathrm{~b}: 0\}\rangle$, the element: $q \otimes !^{\mathrm{a}} \neg r$.

Note that $\Pi$ contains a finite number of elements.

Definition 8 (encoding the halting problem). If $\Gamma$ is $A_{1}, \ldots, A_{n}$, then let $?^{u} \Gamma$ stand for $?^{u} A_{1}, \ldots, ?^{u} A_{n}$. The encoding of the halting problem for the $2 R M$ from the initial configuration $c_{0}=\left\langle q_{0}, v_{0}\right\rangle$ is the $M S E L_{\Xi}$ sequent $\vdash ?^{\infty} \Pi, \mathcal{E}\left(c_{0}\right)$.

Theorem 9. If the $2 R M$ halts from $c_{0}$, then $\vdash_{\Xi} ?^{\infty} \Pi, \mathcal{E}\left(c_{0}\right)$ is derivable.

Proof. We will show that if $c=\left\langle q_{1}, v_{1}\right\rangle \stackrel{i}{\longrightarrow}\left\langle q_{2}, v_{2}\right\rangle=d$ (for some $i$ ), then the following MSEL $\Xi$ rule is derivable:

$$
\frac{\vdash ?^{\infty} \Pi, \mathcal{E}(d)}{\vdash ?^{\infty} \Pi, \mathcal{E}(c)}
$$

This is largely immediate by inspection. Here are three representative cases.

- The case of $i=$ incra: it must be that $v_{2}(\mathrm{a})=v_{1}(\mathrm{a})+1$ and $v_{2}(\mathrm{~b})=v_{1}(\mathrm{~b})$, so $\mathcal{E}(d)=\mathcal{E}(c) \backslash$ $\left\{\neg q_{1}\right\}, \neg q_{2}, ?^{\mathrm{a}}$ ra. Moreover, $q_{1} \otimes\left(\neg q_{2} \not \gamma\right.$ ? $\left.^{\mathrm{a}} \neg \mathrm{ra}\right) \in \Pi$. So:

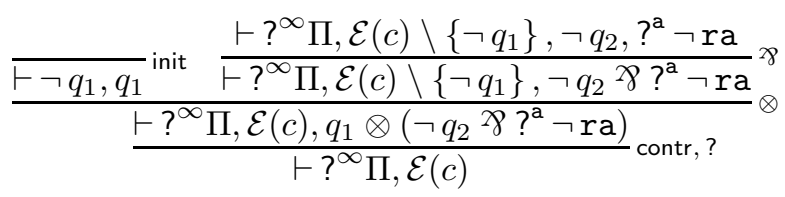

The cases for incrb, decra, and decrb are similar.

- The case of $i=$ isza: it must be that $v_{2}(\mathrm{a})=v_{1}(\mathrm{a})=0$ and $v_{2}(\mathrm{~b})=v_{1}(\mathrm{~b})$. Hence, $\mathcal{E}(d)=\mathcal{E}(c) \backslash$ $\left\{\neg q_{1}\right\}, \neg q_{2}$ and $?^{\mathrm{a}} \mathrm{ra} \notin \mathcal{E}(c) \cup \mathcal{E}(d)$. Moreover, $q_{1} \otimes !^{\mathrm{b}} \neg q_{2} \in \Pi$. So:

$$
\frac{\frac{\vdash \neg q_{1}, q_{1}}{i n i t} \frac{\vdash ?^{\infty} \Pi, \mathcal{E}(c) \backslash\left\{\neg q_{1}\right\}, \neg q_{2}}{\vdash ?^{\infty} \Pi, \mathcal{E}(c) \backslash\left\{\neg q_{1}\right\}, !^{\mathrm{b}} \neg q_{2}}}{\frac{\vdash ?^{\infty} \Pi, \mathcal{E}(c), q_{1} \otimes !^{\mathrm{b}} \neg q_{2}}{\vdash ?^{\infty} \Pi, \mathcal{E}(c)}} \otimes
$$

The instance of ! is justified because $\mathrm{b} \leq \infty$ and $\mathrm{b} \leq \mathrm{b}$, and there are no ?-formulas labeled a or non-? formulas in the sequent. The case of iszb is similar.

- The case of $i=$ halt. Here, we know that $q_{1} \otimes \neg \mathrm{h} \in \Pi$, so:

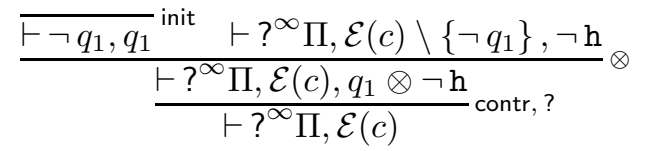

Now, as long as there are any occurrences of $?^{\mathrm{a}} \mathrm{ra}$ or $?^{\mathrm{a}} \mathrm{rb}$ in $\mathcal{E}(c)$, we can apply one of the decrementing rules $\mathrm{h} \otimes !^{\mathrm{a}} \mathrm{ra} \otimes \neg \mathrm{h}$ or $\mathrm{h} \otimes !^{\mathrm{b}} \mathrm{rb} \otimes \neg \mathrm{h} \in \Pi$. The general case looks something like this, where $\Delta_{\mathrm{ra}}=\{\neg \mathrm{ra}, \ldots, \neg \mathrm{ra}\}$ and $\Delta_{\mathrm{rb}}=\{\neg \mathrm{rb}, \ldots, \neg \mathrm{rb}\}$.

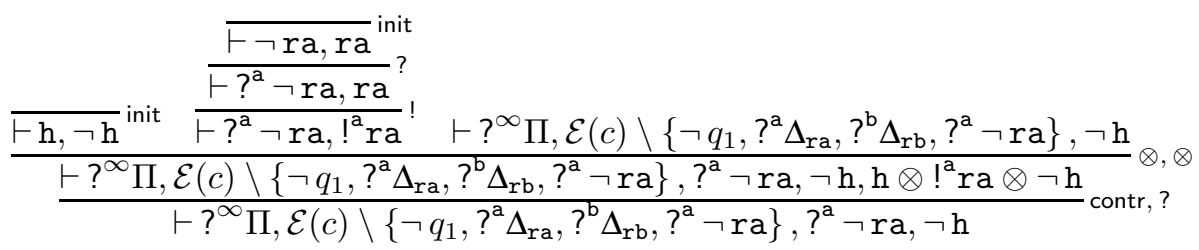




$$
\begin{aligned}
& \frac{\left(\vec{u} \in U_{\Sigma}\right)}{\vdash_{\Sigma} ?^{\vec{u}} \vec{A}, \neg a,[a]}[\text { init }] \quad \frac{\left(\vec{u} \in U_{\Sigma}\right) \vdash_{\Sigma} ?^{\vec{u}} \vec{A}, \Omega_{1},[B] \quad \vdash_{\Sigma} ?^{\vec{u}} \vec{A}, \Omega_{2},[C]}{\vdash_{\Sigma} ?^{\vec{u}} \vec{A}, \Omega_{1}, \Omega_{2},[B \otimes C]}[\otimes] \frac{\left(\vec{u} \in U_{\Sigma}\right)}{\vdash_{\Sigma} ?^{\vec{u}} \vec{A},[1]}[1]
\end{aligned}
$$

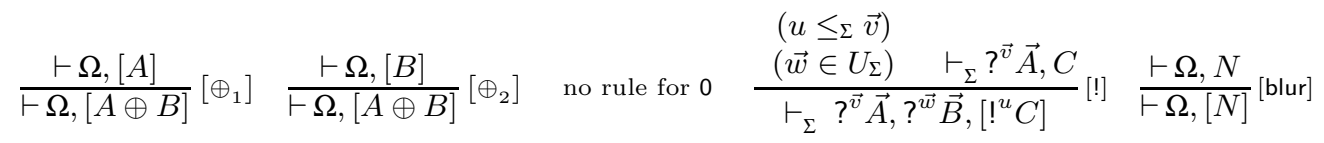

Rules $\ngtr, \perp, \&, \top$ shared with the unfocused system

$$
\frac{\vdash \Omega,[P]}{\vdash \Omega, P} \text { decide } \quad \frac{\left(u \notin U_{\Sigma}\right) \vdash_{\Sigma} \Omega,[A]}{\vdash_{\Sigma} \Omega, ?^{u} A} \text { Idecide } \quad \frac{\left(u \in U_{\Sigma}\right) \vdash_{\Sigma} \Omega, ?^{u} A,[A]}{\vdash_{\Sigma} \Omega, ?^{u} A} \text { udecide }
$$

Figure 2: Inference rules for a focused sequent calculus formulation of SEL.

There is a symmetric case for contracting the $\mathrm{h} \otimes !^{\mathrm{b}} \mathrm{rb} \otimes \neg \mathrm{h}$. Eventually, the right branch just becomes $\vdash ?^{\infty} \Pi, \neg \mathrm{h}$, at which point we have:

$$
\begin{aligned}
& \overline{\vdash 1}^{1} \\
& \vdots \text { weak } \\
& \frac{\frac{\vdash \mathrm{h}, \neg \mathrm{h}}{} \text { init } \frac{\vdash ?^{\infty} \Pi, 1}{\vdash ?^{\infty} \Pi, !^{\infty} 1} !}{\frac{\vdash ?^{\infty} \Pi, \neg \mathrm{h}, \mathrm{h} \otimes !^{\infty} 1}{\vdash ?^{\infty} \Pi, \neg \mathrm{h}} \text { contr, ? }}
\end{aligned}
$$

\section{Adequacy of the Encoding via Focusing}

By the contrapositive of theorem $\underline{9}$, if the sequent $\vdash_{\Xi} ?^{\infty} \Pi, \mathcal{E}\left(c_{0}\right)$ is not derivable, then the $2 R M$ does not halt from $c_{0}$. This gives half of the reduction. For the converse of theorem $\underline{9}$, we need to show how to recover a halting trace by searching for proofs of a $M S E L \Xi$ encoding of a halting problem. The best way to do this is to build a focused proof which will have the derived inference rules in the above proof as the only possible synthetic rules, in a sense made precise below. We will begin by sketching the focused proof system for SEL that is sound and complete for the unfocused system of figure $\underline{1}$, and then show how the synthetic rules for the encoding are in bijection for all instructions (with a small correction needed for halt).

Focusing is a general technique to restrict the non-determinism in a cut-free sequent proof system. Though originally defined for classical linear logic in [1], it is readily extended to many other logics $[\underline{4}, \underline{5}, \underline{10}]$. This section sketches the basic design of a focused version of the rules of figure $\underline{1}$, and omits most of the meta-theoretic proofs of soundness and completeness, for which the general proof techniques are by now well known [또 $\underline{8}, \underline{15}$. To keep things simple, we will define a focused calculus by adding to the unfocused system a new kind of focused sequent, $\vdash \Omega,[A]$, where the formula $A$ is under focus. Contexts written with $\Omega$, which we call neutral contexts, can contain only positive formulas, atoms, negated atoms, and ?-formulas. The rules of the focused proof system for $S E L$ are depicted in figure $\underline{2}$.

Focused sequents are created - reading from conclusion upwards to premises - from unfocused sequents with neutral contexts by means of the rules decide, Idecide, or udecide. In a focused sequent, only the formula under focus can be principal, and the focus persists on the immediate subformulas of this formula in the premises, with the exception of the rule [!]. In the base case, for [init], the focused atom must find its negation in the context, while all formulas in the context must be ?-formulas with unbounded labels. When the focused formula is negative, the focus is released with the [blur] rule, at which point any of the unfocused rules $\{\not>, \perp, \&, \top\}$ of figure 1 can be used to decompose the formula and its descendants further. Eventually, when there are no more negative descendants - i.e., the whole context has the form $\Omega$-a new focused phase is launched again and the cycle repeats. Note that the structural rules contr and weak of the unfocused calculus are removed in the focused system. Instead, weakening is folded into [init], [!], and [1], and contraction is folded into [ $\otimes$ ] and udecide. The rules contr and weak remain admissible for either sequent form in the focused calculus.

Theorem 10. The SEL sequent $\vdash \Gamma$ is provable in the unfocused system of figure 1 if and only if it is provable in the focused system of figure $\underline{2}$.

Proof. Straightforward adaptation of existing proofs of the soundness and completeness of focusing, such as $[\underline{4}$ $\underline{8}, \underline{15}$. An instance for $S E L$ can be found in [10, chapter 5]. 
Theorem 11. The $2 R M$ halts from $c_{0}$ if $\vdash_{\Xi} ?^{\infty} \Pi, \mathcal{E}\left(c_{0}\right)$ is derivable.

Proof. We will show instead that the $2 R M$ halts from $c_{0}$ if the sequent $\vdash_{\Xi} ?^{\infty} \Pi, \mathcal{E}\left(c_{0}\right)$ is derivable in the focused calculus, and we will moreover extract the halting trace from such a focused proof. The required result will then follow immediately from theorem 10 , since any provable SEL sequent has a focused proof.

Let a focused proof of $\vdash_{\Xi} ?^{\infty} \Pi, \mathcal{E}(c)$ (for $c=\langle q, v\rangle$ ) be given. We proceed by induction on the lowermost instance of udecide in this proof. Note that the MSEL $\Xi$ context $?^{\infty} \Pi, \mathcal{E}(c)$ is neutral; moreover, all the elements of $\mathcal{E}(c)$ are either negated atoms or ?-prefixed negated atoms with bounded labels. So, the only rules of the focusing system that apply to this sequent are Idecide or udecide. However, if we use Idecide, then the premise becomes unprovable, as there is no way to remove an occurrence of $\neg \mathrm{ra}$ or $\neg \mathrm{rb}$ from a context that also contains $\neg q$. Thus, the only possible rule will be an instance of udecide, with the focused formula in the premise being one of the $\Pi$. First, consider the case where the focused formula does not contain h, i.e., it corresponds to one of the instructions in $I \backslash\{$ halt $\}$. In each of these cases, the focused phase that immediately follows is deterministic. As a characteristic case, suppose the focused formula is $q \otimes !^{\mathrm{b}} \neg r$; then we have:

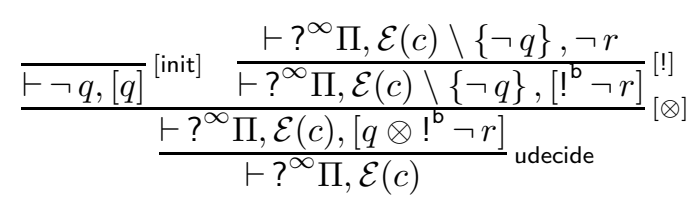

The right premise is now itself neutral and an encoding of a different configuration. We can appeal to the inductive hypothesis to find a halting trace for it, to which we can prepend the instruction isza to get the halting trace from $c$. A similar argument can be used for the other instructions in $I \backslash\{$ halt $\}$.

This leaves just the formulas involving $\mathrm{h}$ for the lowermost udecide. We cannot select any formula but $q \otimes \neg \mathrm{h}$ from $\Pi$, for the derivation would immediately fail because $\mathrm{h} \notin Q$ and there is no $\neg \mathrm{h}$ in $\mathcal{E}(c)$ to use with [init]. So, as the formula selected is $q \otimes \neg \mathrm{h}$, we have:

$$
\frac{\overline{\vdash \neg q,[q]}_{[\text {init] }} \frac{\vdash ?^{\infty} \Pi, \mathcal{E}(c) \backslash\{\neg q\}, \neg \mathrm{h}}{\vdash ?^{\infty} \Pi, \mathcal{E}(c) \backslash\{\neg q\},[\neg \mathrm{h}]} \text { [blur] }}{\frac{\vdash ?^{\infty} \Pi, \mathcal{E}(c),[q \otimes \neg \mathrm{h}]}{\vdash ?^{\infty} \Pi, \mathcal{E}(c)}} \otimes
$$

The context of the right premise is now neutral, so the only rule that applies to it is udecide. A simple nested induction will show that sequents of this form $\vdash ?^{\infty} \Pi, \mathcal{E}(c) \backslash\{\neg q\}, \neg \mathrm{h}$ are always derivable in the focused calculus. Therefore, the trace that corresponds to the configuration $c$ is just the singleton halt.

Corollary 12. The derivability of $M S E L_{\Xi}$ sequents is recursively unsolvable.

Proof. Directly from theorems $\underline{4}, \underline{9}$ and $\underline{11}$.

\section{Directly Encoding $M A L L$}

Since $M S E L \Xi$ is Turing-equivalent, it can obviously be used to simulate a theorem prover that implements a complete search procedure for MAELL. Thus, in an indirect fashion, we see that additive behavior can be encoded using multiplicatives and subexponentials alone. In this section we will give a more direct demonstration of this ability by showing how to simulate propositional MALL in a propositional MSEL.

We use the propositional version to keep the result as strong as possible. As a price, the encoding needs to be specialized to every subformula of the endsequent, and is therefore exponentially bigger than the MALL sequent we start with. If we were to use a first-order variant of MSEL with the same signature, then our encoding would be polynomial in the size of the MALL sequent, since the theory of MALL would be of constant size. This is indeed the technique used by many of the encodings of various proof systems in SEL [14].

Definition 13. Let $\Upsilon$ stand for the signature $\langle\{\infty, 1, r, \operatorname{lr}, \operatorname{lin}\},\{\infty\}, \leq\rangle$ where $\leq$ is the reflexive-transitive closure of $\leq_{0}$ defined by $\mathrm{l} \leq_{0} \infty, \mathrm{r} \leq_{0} \infty$, $\operatorname{lr} \leq_{0} \mathrm{l}$, $\operatorname{lr} \leq_{0} \mathrm{r}$ and $\operatorname{lin} \leq_{0} \infty$. In other words, $\leq$ has the following lattice.

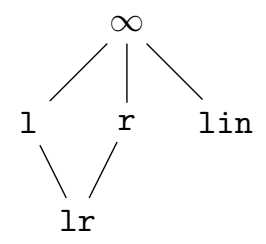


Recall that MALL formulas are built from literals (atoms or negated atoms) and the connectives $\{\otimes, 1, \not \supset, \perp, \oplus, 0, \&, \top\}$. As this is a sub-language of SEL, we use the same inference system as in Figure 1 . We will build an MSEL $\Upsilon$ sequent whose MSEL proofs are in bijection to the MALL proofs of $\vdash \Gamma$.

Notation 14. We write $A \varepsilon B$ to indicate that $A$ is a subformula of $B$. Likewise $A \varepsilon \Gamma$ means that $A \varepsilon B$ for some $B \in \Gamma$.

Definition 15 (atomic formulas). If $\Gamma$ is a multiset of MALL formulas, then we add the following atomic formulas to the collection of atoms used in the MSEL $\Upsilon$ encoding of $\Gamma$.

- The atom rule to represent the conclusions and premises of every MALL inference rule.

- For every $C \varepsilon \Gamma$, the atom $\mathrm{f}_{C}$ to represent an occurrence of $C$. If $\Gamma$ is the multiset $\left\{A_{1}, \ldots, A_{n}\right\}$, then we write $\mathbf{f}_{\Gamma}$ to stand for the multiset $\left\{\mathbf{f}_{A_{1}}, \ldots, \mathbf{f}_{A_{n}}\right\}$.

- For every $A \oplus B \varepsilon \Gamma$, the atom $\operatorname{ch}_{A, B}$ to represent the choice between $A$ and $B$.

- The atoms cp, restl and restr to represent stages in the copying procedure for contexts.

- The atom gc to represent stages in the deletion procedure for contexts.

We assume that none of these atomic formulas occurs as a subformula of $\Gamma$.

We will use the above subexponential signature and collection of atomic formulas to encode the MALL inference system specialized to the endsequent $\vdash \Gamma$. This encoding will be in the form of a theory $\Theta_{\Gamma}$ that will be a collection of formulas with !s and ?s interspersed to place and check occurrences.

Definition 16 (MALL encoding theory). If $\Gamma$ is a multiset of $M A L L$ formulas, then the theory $\Theta_{\Gamma}$ is made up of the following elements.

- For every atom $a \varepsilon \Gamma$,

$$
\text { rule } \otimes !^{\text {in }} \neg \mathrm{f}_{a} \otimes !^{\text {lin }} \neg \mathrm{f}_{\neg a} .
$$

- For every $A \otimes B \varepsilon \Gamma$, the formula:

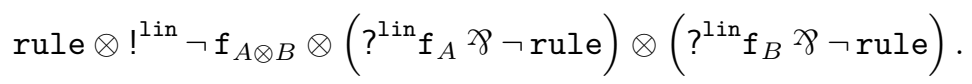

- If $1 \varepsilon \Gamma$, then:

$$
\text { rule } \otimes !^{\text {lin }} \neg f_{1} .
$$

- For every $A \not \supset B \varepsilon \Gamma$, the formula:

$$
\text { rule } \otimes !^{l \text { in }} \neg f_{A} \gamma{ }_{B} \otimes\left(?^{1 \text { in }} f_{A} \not \gamma ?^{l i n} f_{B} \not \neg \neg \text { rule }\right) .
$$

- If $\perp \varepsilon \Gamma$, then:

$$
\text { rule } \otimes !^{\text {lin }} \neg f_{\perp} \otimes \neg \text { rule. }
$$

- For every $A \oplus B \varepsilon \Gamma$, the formulas:

$$
\begin{aligned}
& \text { rule } \otimes !^{l i n} \neg f_{A \oplus B} \otimes \neg \mathrm{ch}_{A, B}, \\
& \operatorname{ch}_{A, B} \otimes\left(?^{l i n} f_{A} \not \neg \text { rule }\right), \\
& \operatorname{ch}_{A, B} \otimes\left(?^{l i n} f_{B} \not \neg \neg \text { rule }\right) .
\end{aligned}
$$

- For every $A \& B \varepsilon \Gamma$, the formulas:

$$
\begin{aligned}
& \text { rule } \otimes !^{l \text { in }} \neg \mathrm{f}_{A \& B} \otimes \neg \mathrm{cp}, \\
& \text { restl } \otimes !^{l \text { in }}\left(?^{\text {lin }} \mathrm{f}_{A} \not \neg \text { rule }\right), \\
& \operatorname{restr} \otimes !^{l \text { in }}\left(?^{\text {lin }} \mathrm{f}_{B} \not \neg \neg \text { rule }\right) .
\end{aligned}
$$


- To implement copying, the following formula for every $C \varepsilon \Gamma$ :

$$
\mathrm{cp} \otimes !^{\text {lin }} \neg \mathrm{f}_{C} \otimes\left(?^{1} \mathrm{f}_{C} \not \gamma ?^{\mathrm{r}} \mathrm{f}_{C} \not \gamma \neg \mathrm{cp}\right) .
$$

To detect when copying is finished, the additional formula:

$$
\mathrm{cp} \otimes !^{\operatorname{lr}}\left(!^{l} \neg \text { restl } \otimes !^{\mathrm{r}} \neg \text { restr }\right) .
$$

- Once copying is finished, to implement the transferal of formulas from the temporary subexponential labels 1 or $r$ to the main label lin, the following formulas for every $C \varepsilon \Gamma$ :

$$
\begin{aligned}
& \text { restl } \otimes !^{1} \neg \mathrm{f}_{C} \otimes\left(?^{l \text { in }} \mathrm{f}_{C} \not \neg \neg \text { restl }\right), \\
& \text { restr } \otimes !^{\mathrm{r}} \neg \mathrm{f}_{C} \otimes\left(?^{l \text { in }} \mathrm{f}_{C} \not \gamma \neg \text { restr }\right) .
\end{aligned}
$$

Note that the transferal process ends with one of the latter two steps in the theory elements for $A \& B$ above.

- If $\top \varepsilon \Gamma$, then the following two formulas to initiate and terminate deletion:

$$
\text { rule } \otimes !^{l i n} \neg f_{\top} \otimes \neg \mathrm{gc}, \quad \mathrm{gc} .
$$

- To implement deleting the context during a proof of $T$, the following formula for every $C \varepsilon \Gamma$ :

$$
\mathrm{gc} \otimes !^{\text {lin }} \neg \mathrm{f}_{C} \otimes \neg \mathrm{gc} .
$$

Note: since $\Gamma$ has finitely many subformulas, it must be that $\Theta_{\Gamma}$ is finite.

This brings us to the encoding of MALL contexts.

Definition 17 (encoding $M A L L$ contexts). If $\Gamma$ is a multiset of $M A L L$ formulas, then $\mathcal{E}(\Gamma)$ stands for the multiset

$$
?^{\infty} \Theta_{\Gamma},\left\{?^{\operatorname{lin}} \mathrm{f}_{C}: C \in \Gamma\right\}, \neg \text { rule. }
$$

We will now prove the following adequacy theorem.

Theorem 18. If $\Gamma_{0}$ is a multiset of MALL formulas, then $\vdash_{M A L L} \Gamma_{0}$ if and only if $\vdash_{M S E L \Upsilon} \mathcal{E}\left(\Gamma_{0}\right)$.

Proof. Let us first show that the encoding can simulate MALL proofs, i.e., if $\vdash_{\text {MALL }} \Gamma_{0}$ then $\vdash_{\text {MSEL }} \mathcal{E}\left(\Gamma_{0}\right)$. We will establish this by showing that all the inference rules of MALL are simulated by the encoding, i.e., for every $M A L L$ inference rule of the form

$$
\frac{\vdash \Gamma_{1} \cdots \quad \vdash \Gamma_{n}}{\vdash \Gamma_{0}}
$$

there is a derived MSEL $\Upsilon$ inference rule for

$$
\frac{\vdash \mathcal{E}\left(\Gamma_{1}\right) \cdots \quad \vdash \mathcal{E}\left(\Gamma_{n}\right)}{\vdash \mathcal{E}\left(\Gamma_{0}\right)}
$$

Note that this proof only makes sense if the MALL inference system has the subformula property-otherwise, $\mathcal{E}\left(\Gamma_{1}\right), \ldots, \mathcal{E}\left(\Gamma_{n}\right)$ would not be fragments of $\mathcal{E}\left(\Gamma_{0}\right)$ - which is the case for us since our proof system (Figure 1$)$ is cut-free. There are the following cases.

- Case of init: here, we know that

$$
\begin{aligned}
\mathcal{E}(a, \neg a)= & ?^{\infty}\left(\text { rule } \otimes !^{\text {lin }} \neg \mathrm{f}_{a} \otimes !^{\text {lin }} \neg \mathrm{f}_{\neg a}\right), \\
& ?^{l i{ }_{f_{a}},} ?^{l \text { in }} \mathrm{f}_{\neg a}, \neg \text { rule. }
\end{aligned}
$$

so:

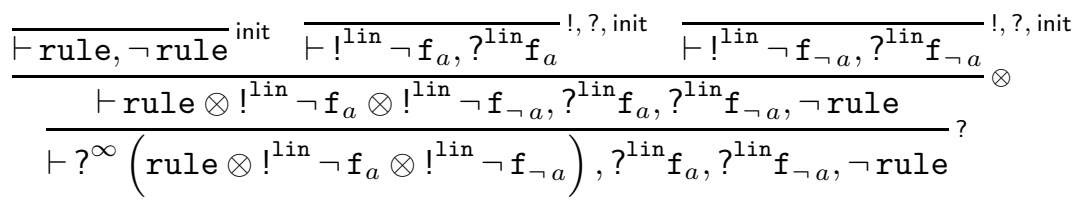


- Case of $\otimes$ : we know that:

$$
\mathcal{E}(\Gamma, \Delta, A \otimes B)=?^{\infty} \Theta_{\Gamma, \Delta, A \otimes B}, ?^{\text {lin }} \mathrm{f}_{\Gamma}, ?^{l i n} \mathrm{f}_{\Delta}, ?^{l i n} f_{A \otimes B}, \neg \text { rule. }
$$

where

$$
\Theta_{\Gamma, \Delta, A \otimes B} \ni\left(\text { rule } \otimes !^{l i n} \neg f_{A \otimes B} \otimes\left(?^{l i n} f_{A} \not \gamma \neg \text { rule }\right) \otimes\left(?^{l i n} f_{B} \not \supset \neg \text { rule }\right)\right) .
$$

Moreover, $\Theta_{\Gamma, \Delta, A \otimes B}$ is the same set of formulas as $\Theta_{\Gamma, A}, \Theta_{\Delta, B}, R$, so the latter is related to the former by a sequence of contractions. So, we have:

$$
\frac{\vdash ?^{\infty} \Theta_{\Gamma, A}, ?^{l i n} f_{\Gamma}, ?^{l i n} f_{A}, \neg \operatorname{rule} \vdash ?^{\infty} \Theta_{\Delta, B}, ?^{l i n} f_{\Delta}, ?^{l i n} f_{B}, \neg \text { rule }}{\vdash ?^{\infty} \Theta_{\Gamma, \Delta, A \otimes B}, ?^{l i n} f_{\Gamma}, ?^{l i n} f_{\Delta}, ?^{l i n} f_{A \otimes B}, \neg \text { rule. }} \text { contr }, ?, \otimes, !, \text { init }
$$

where the two additional closed branches are not shown.

- The case of 1 is a trivial analogue of the previous case.

- The cases of $\not 8$ and $\perp$ are completely straightforward.

- The case of $\oplus$ : we know that

$$
\mathcal{E}(\Gamma, A \oplus B)=?^{\infty} \Theta_{\Gamma, A \oplus B}, ?^{l i n} \mathrm{f}_{\Gamma}, ?^{l \text { in }} \mathrm{f}_{A \oplus B}, \neg \text { rule. }
$$

where

$$
\begin{aligned}
& \Theta_{\Gamma, A \oplus B} \supseteq \operatorname{rule} \otimes !^{l i n} \neg f_{A \oplus B} \otimes \neg \operatorname{ch}_{A, B}, \\
& \operatorname{ch}_{A, B} \otimes\left(?^{l i n} f_{A} \not \neg \neg \text { rule }\right), \\
& \operatorname{ch}_{A, B} \otimes\left(?^{l i n} f_{B} \not \neg \neg \text { rule }\right) .
\end{aligned}
$$

Note that both $\Theta_{\Gamma, A}$ and $\Theta_{\Gamma, B}$ are reachable from $\Theta_{\Gamma, A \oplus B}$ by a sequence of weakenings. So, we have the following derivation as one possibility, where many irrelevant details and closed branches are omitted for lack of space.

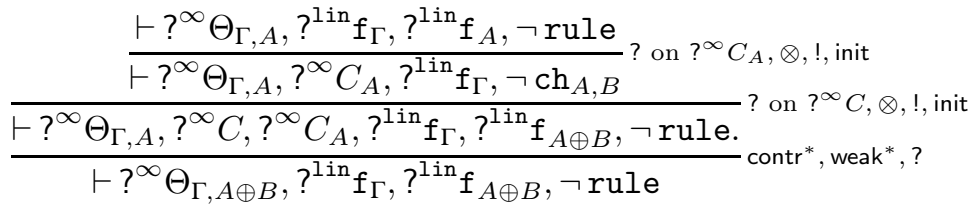

The other possibility is that $C_{A}$ is weakened and $C_{B}$ is contracted, which is symmetric.

- Case of \&: we know that:

$$
\mathcal{E}(\Gamma, A \& B)=?^{\infty} \Theta_{\Gamma, A \& B}, ?^{l i n} \mathrm{f}_{\Gamma}, ?^{l i n} \mathrm{f}_{A \& B}, \neg \text { rule }
$$

where:

$$
\begin{aligned}
& \Theta_{\Gamma, A \& B} \supseteq \mathrm{rule} \otimes !^{\mathrm{lin}} \neg \mathrm{f}_{A \& B} \otimes \neg \mathrm{cp}, \\
& \left\{\mathrm{cp} \otimes !^{\text {lin }} \neg \mathrm{f}_{C} \otimes\left(?^{\mathrm{l}} \mathrm{f}_{C} \not \gamma ?^{\mathrm{r}} \mathrm{f}_{C} \not \gamma \neg \mathrm{cp}\right): C \varepsilon \Gamma_{0}\right\}, \\
& \mathrm{cp} \otimes !^{\mathrm{lr}}\left(!^{\mathrm{l}} \neg \text { restl } \otimes !^{\mathrm{r}} \neg \text { restr }\right), \\
& \left\{\text { restl } \otimes !^{1} \neg \mathrm{f}_{C} \otimes\left(?^{\text {lin }} \mathrm{f}_{C} \not \neg \neg \text { restl }\right): C \varepsilon \Gamma_{0}\right\} \text {, } \\
& \left\{\text { restr } \otimes !^{r} \neg f_{C} \otimes\left(?^{l i n} f_{C} \not \gamma \neg \text { restr }\right): C \varepsilon \Gamma_{0}\right\} \text {. } \\
& \text { restl } \otimes !^{\text {lin }}\left(?^{\text {lin }_{f_{A}}} \not \neg \neg \text { rule }\right) \text {, } \\
& \text { restr } \otimes !^{\text {lin }}\left(?^{\text {lin }} \mathrm{f}_{B} \not \gamma \neg \text { rule }\right) \text {. }
\end{aligned}
$$

Moreover, both $\Theta_{\Gamma, A}$ and $\Theta_{\Gamma, B}$ are related to $\Theta_{\Gamma, A \& B}$ by contraction and weakening. The derivation begins as follows, where we now also elide the rule names for lack of space, but note just which of $R_{1}, \ldots, R_{7}$ 
above were principal.

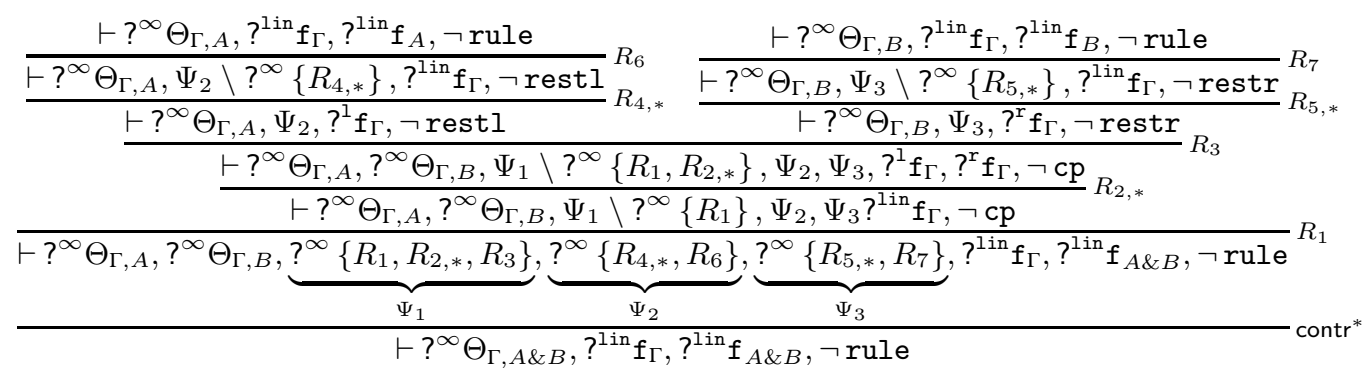

There are three crucial points. The first is the inference corresponding to $R_{3}$ : this rule requires all the subexponential labels in the context to be above $l r$, which is the case since both $l r \leq 1$ and $l r \leq r$. This rule could not be applied any earlier (lower) because lin $\not \leq l r$; it had to wait until the $R_{2, *}$ rules would transfer all the $?^{\text {lin }}$ formulas. Once this outer test succeeds, we apply the $\otimes$ rule, and have two inner tests; the left test checks for the emptiness of $r$ since $l \not \leq r$, and the right test does the converse. This guarantees that all the $?^{1}$ formulas are indeed sent to the left branch, and the $?^{\mathrm{r}}$ formulas to the right branch. The other two crucial points are the applications corresponding to $R_{6}$ and $R_{7}$. In each case, the $!^{\text {lin }}$ guard checks that the 1 or $\mathrm{r}$ formulas (as applicable) are absent since lin $\not \leq l$ and $\operatorname{lin} \not \leq \mathrm{r}$. Neither of these rules could have been applied any earlier, while there were still $?^{1}$ or $?^{\mathrm{r}}$ formulas left to transfer back to lin.

- Finally, the case of $T$ : we know that:

$$
\mathcal{E}(\Gamma, \top)=?^{\infty} \Theta_{\Gamma, \top}, ?^{\text {lin }} \mathrm{f}_{\Gamma}, ?^{1 \text { in }} \mathrm{f}_{\top}, \neg \text { rule. }
$$

where:

$$
\begin{aligned}
\Theta_{\Gamma, \mathrm{T}} \supseteq \operatorname{rule} \otimes !^{\text {lin }} \neg \mathrm{f}_{\mathrm{T}} \otimes \neg \mathrm{gc}, \\
\quad\left\{\mathrm{gc} \otimes !^{1 \mathrm{in}} \neg \mathrm{f}_{C} \otimes \neg \mathrm{gc}: C \varepsilon \Gamma_{0}\right\} .
\end{aligned}
$$

We have:

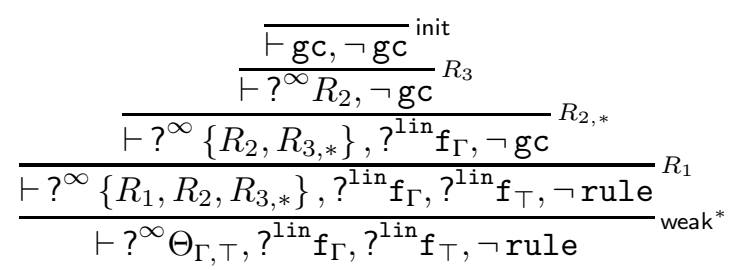

As in the previous case, the key rule $R_{2}$ could not be applied any earlier (lower) if there were any $?^{\text {lin }}$ formulas left, since lin is a linear subexponential.

In the reverse direction, we follow the strategy of Theorem 11 and show that the above derived inferences are the only possible ones in a focused proof system. The argument is fairly straightforward: in each case, the use of the atoms rule, ch, cp, restl, restr, and gc guarantee that only the relevant formulas from $\Theta_{\Gamma_{0}}$ can be decided on; any other decision would immediately fail. The $f$ atoms further limit the selection of the elements of $\Theta_{\Gamma_{0}}$ to those that are the encoding of the correct principal formulas. As already argued above, the computations in $\oplus, \&$, and $T$ are carefully guarded with !s to prevent any interleaving and to ensure the correct distribution of the linear resources; any other distribution would lead to a failure within the same phase of focusing. Thus, from a focused $M S E L_{\Upsilon}$ proof of $\vdash \mathcal{E}\left(\Gamma_{0}\right)$, if we abstract the proof to keep only those sequents that contain $\neg$ rule, and then erase everything except the f-atoms, we would get a proof tree that is isomorphic to a MALL proof of $\vdash \Gamma_{0}$.

\section{Perspectives}

The conclusion of Section $\underline{4}$ is that it is not the additives that make MAELL undecidable but rather the fact that the additives can be used to implement a kind of limited test for a portion of the linear resources. Thus, if we have access to such tests using other logical means, then the decision problem can still be undecidable. Section $\underline{5}$ further shows that if we have such alternative tests, then we can in fact implement the additive 
connectives. Thus, these alternative tests are in fact stronger than the additive connectives. A natural question then is: are the mechanisms provided by subexponentials strictly stronger than additives? Once again, there is an indirect negative answer in the sense of implementing a complete theorem prover for a MSEL using the Turing-equivalence of MAELL. It would be good to have a more direct encoding of any MSEL in MAELL, but it is not obvious how to construct such encodings.

We submit the two technical theorems in Sections $\underline{4}$ and $\underline{5}$ as arguments for a philosophical view of $M S E L$ as a logic of ordinary computers. A standard computer-with a Von Neumann architecture, say, but this is not important - is manifestly able to maintain a bank of counters and to observe and act upon the value of any particular counter. It is unnatural, though, to allow such a computer to take computational steps that depend on a different computation in a parallel reality that is identical to the present reality in all but a principal aspect. The encoding of Minsky machines in MAELL requires such nonstandard computationsl steps. On the other hand, every action that is expressible in MSEL corresponds to a natural single-threaded computational feature: $\otimes$ corresponds to separation, $\gamma$ to concurrency, ? to placement or context switching, and ! to waiting or sequentialization.

There are a number of other logical systems that provide some kind of staged computation or provide mechanisms for testing for properties of a subset of linear resources. Probably the most widely known is the computational monad approach pioneered by Concurrent LF [16], which was then implemented in the Lollimon logic programming language [7]. This idea can be seen as extending linear logic with a lax modality and then giving it an operational mode of computation up to quiescence. As far as we are aware, the decidability of the propositional fragment of this logic is still open. In the non-linear world there are far too many techniques for incorporating staged computations to survey in this article. We simply note that most propositional non-linear modal logics turn out to be decidable.

Any MSEL with only linear subexponentials will be decidable because contraction is the only way to make a proof infinitely deep. This work leaves open the questions of decidability of an arbitrary MSEL with a twoelement signature where at least one subexponential is unbounded. Such logics lie between $M S E L_{\Xi}$ and $M E L L$ itself and may have easier decision problem than the latter.

Finally, this undecidability result should be taken as a word of caution for the increasingly popular uses of SEL as a logical framework for the encodings of other systems $[\underline{12}, \underline{13}$, e.g.]. If one is to avoid encoding a decidable problem in terms of an undecidable one, subexponentials must be used very carefully.

\section{Acknowledgements}

We thank Giselle Reis for many fruitful discussions about encoding proof systems in SEL, and Anupam Das for his encouragement to finish the material in Section $\underline{5}$.

\section{References}

[1] J.-M. Andreoli. Logic programming with focusing proofs in linear logic. J. of Logic and Computation, $2(3): 297-347,1992$.

[2] K. Chaudhuri. Classical and intuitionistic subexponential logics are equally expressive. In CSL 2010: Computer Science Logic, volume 6247 of Lecture Notes in Computer Science, pages 185-199. Springer, Aug. 2010 .

[3] K. Chaudhuri. Undecidability of multiplicative subexponential logic. In S. Alves and I. Cervesato, editors, Proceedings LINEARITY 2014, volume 176, pages 1-6. EPTCS, 2014.

[4] K. Chaudhuri, F. Pfenning, and G. Price. A logical characterization of forward and backward chaining in the inverse method. J. of Automated Reasoning, 40(2-3):133-177, Mar. 2008.

[5] C. Liang and D. Miller. Focusing and polarization in linear, intuitionistic, and classical logics. Theoretical Computer Science, 410(46):4747-4768, 2009.

[6] P. Lincoln, J. Mitchell, A. Scedrov, and N. Shankar. Decision problems for propositional linear logic. Annals of Pure and Applied Logic, 56:239-311, 1992.

[7] P. López, F. Pfenning, J. Polakow, and K. Watkins. Monadic concurrent linear logic programming. In P. Barahona and A. P. Felty, editors, Proceedings of the rth International ACM SIGPLAN Conference on Principles and Practice of Declarative Programming (PPDP), pages 35-46. ACM, 2005.

[8] D. Miller and A. Saurin. From proofs to focused proofs: a modular proof of focalization in linear logic. In J. Duparc and T. A. Henzinger, editors, CSL 2007: Computer Science Logic, volume 4646 of Lecture Notes in Computer Science, pages 405-419. Springer, 2007. 
[9] M. Minsky. Recursive unsolvability of Post's problem of 'tag' and other topics in the theory of Turing machines. Annals of Mathematics, 74(3):437-455, 1961.

[10] V. Nigam. Exploiting non-canonicity in the sequent calculus. PhD thesis, Ecole Polytechnique, Sept. 2009.

[11] V. Nigam and D. Miller. Algorithmic specifications in linear logic with subexponentials. In ACM SIGPLAN Conference on Principles and Practice of Declarative Programming (PPDP), pages 129-140, 2009.

[12] V. Nigam, C. Olarte, and E. Pimentel. A general proof system for modalities in concurrent constraint programming. In 24th International Conference on Concurrency Theory (CONCUR), volume 8052 of LNCS, pages 410-424. Springer, 2013.

[13] V. Nigam, E. Pimentel, and G. Reis. Specifying proof systems in linear logic with subexponentials. Electronic Notes in Theoretical Computer Sciience, 269:109-123, 2011.

[14] V. Nigam, E. Pimentel, and G. Reis. An extended framework for specifying and reasoning about proof systems. J. of Logic and Computation, 2014.

[15] R. J. Simmons. Structural focalization. ACM Trans. on Computational Logic, 15(3):21, 2014.

[16] K. Watkins, I. Cervesato, F. Pfenning, and D. Walker. A concurrent logical framework I: Judgments and properties. Technical Report CMU-CS-02-101, Carnegie Mellon University, 2003. Revised, May 2003. 